\title{
INTEGRAL MEANS OF CERTAIN CLASS OF ANALYTIC FUNCTIONS
}

\author{
GAO CHUNYI \\ Department of Mathemalıcs \\ Changsha Communications Institute \\ Changsha, Hunan 410076 \\ People's Republic of China
}

(Received June 20, 1991 and in revised form September 3, 1992)

ABSTRACT. In this paper we discuss the following class of functions

$$
S_{\lambda}(\alpha, \beta)=\left\{f(z):\left|\frac{f(z)}{g(z)}-1\right|<\beta\left|\lambda \frac{f(z)}{g(z)}+1\right|, z \in D\right\} \text { where } 0 \leq \lambda \leq 1,0<\beta \leq 1,0 \leq \alpha<1,
$$

and $f(z)=z+\sum_{n=2}^{\infty} a_{n} z^{n}$ is analytic in $D=\{z:|z|<1\}, g(z)$ is a starlike function of order $\alpha$. A subordination about this class is obtained, the integral means of functions in $S_{\lambda}(\alpha, \beta)$ and some extremal properties are studied.

KEY WORDS AND PHRASES. Analytic function, subordination, integral mean, distortion, coefficient inequality.

1991 AMS SUBJECT CLASSIFICATION CODE. 30C45.

\section{INTRODUCTION.}

Let $A$ be the class consisting of all functions $f(z)=z+\sum_{n=2}^{\infty} a_{n} z^{n}$ which are analytic in $D=\{z:|z|<1\}$. Owa [1] has introduced the class $\tilde{S}_{\lambda}(\alpha, \beta)^{n=2}$ If $f(z) \in A$ and there exist: $g(z)=z-\sum_{n=2}^{\infty}\left|b_{n}\right| z^{n} \in S^{*}(\alpha)(0 \leq \alpha<1)$ such that

$$
\left|\frac{f(z)}{g(z)}-1\right|<\beta\left|\lambda \frac{f(z)}{g(z)}+1\right| \quad(0 \leq \lambda \leq 1,0<\beta \leq 1, z \in D)
$$

we say $f(z) \in \tilde{S}_{\lambda}(\alpha, \beta)$. Owa [1] discussed the coefficient estimates of functions in $\tilde{S}_{\lambda}(\alpha, \beta)$. In this paper, we discuss the general case, i.e., the class $S_{\lambda}(\alpha, \beta)$ which is generated by a function

$$
g(z)=z+\sum_{n=2}^{\infty} b_{n} z^{n} \in S^{*}(\alpha) \text {. }
$$

We first gave a subordinate about this class, then we discuss the integral means of functions in $S_{\lambda}(\alpha, \beta)$, from this we can get some extremal properties about $S_{\lambda}(\alpha, \beta)$. We also discuss a subclass of $S_{\lambda}(\alpha, \beta)$.

2. A SUBORDINATION ABOUT $\mathrm{S}_{\lambda}(\alpha, \beta)$.

We say that $g(z)$ is subordinate to $f(z)$ if there exists a function $\omega(z)$ analytic in $D$ satisfying $\omega(0)=0$ and $|\omega(z)|<1$ such that $g(z)=f(\omega(z)) \quad(|z|<1)$. This subordination is denoted by $g(z) \prec f(z)$. About the class $S_{\lambda}(\alpha, \beta)$, we have the following:

THEOREM 2.1. If $f(z) \in S_{\lambda}(\alpha, \beta)$, i.e., there exists a function $g(z) \in S^{*}(\alpha)$ such that the inequality (1.1) holds, then we have

$$
\frac{f(z)}{g(z)} \prec \frac{1+\beta z}{1-\beta \lambda z}=p_{\beta, \lambda}(z) .
$$


PROOF. Let $p(z)=\frac{f(z)}{g(z)}$, then $p(0)=1$. Now we divide the proof into three cases.

CASE (a). Let $\lambda \neq 0, \beta$ and $\lambda$ are not equal to 1 at the same time. Now the inequality (1.1) can be written as $|p(z)-1|<|\beta \lambda p(z)+\beta|$, that is,

$|p(z)|^{2}-2 \operatorname{Rep}(z)+1<\beta^{2} \lambda^{2}|p(z)|^{2}+2 \beta^{2} \lambda \operatorname{Rep}(z)+\beta^{2}$. From this we can get

$$
\left|p(z)-\frac{1-\beta}{1+\beta \lambda}-\frac{\beta(1+\lambda)}{1-\beta^{2} \lambda^{2}}\right|<\frac{\beta(1+\lambda)}{1-\beta^{2} \lambda^{2}} .
$$

Because univalent function $p_{\beta, \lambda}(z)=\frac{1+\beta z}{1-\beta \lambda_{z}}$ maps $D$ onto the disk

$$
\left\{w:\left|w-\frac{1-\beta}{1+\beta \lambda}-\frac{\beta(1+\lambda)}{1-\beta^{2} \lambda^{2}}\right|<\frac{\beta(1+\lambda)}{1-\beta^{2} \lambda^{2}}\right\},
$$

so $p(D) \subset p_{\beta, \lambda}(D)$ and $p(0)=p_{\beta, \lambda}(0)=1$. From the principle of subordination of univalent functions, we have $p(z) \prec p_{\beta, \lambda}(z)$, that is $(2.1)$.

CASE (b). Let $\lambda=0$. Now the inequality (1.1) becomes $|p(z)-1|<\beta$.

Because univalent function $p_{\beta, o}(z)=1+\beta z$ maps $D$ onto the disk $\{w:|w-1|<\beta\}$, so $p(D) \subset p_{\beta, o}(D)$ and $p(0)=p_{\beta, o}(0)=1$. Thus $p(z) \prec p_{\beta, o}(z)$.

$\operatorname{CASE}(\mathrm{c})$. Let $\lambda=\beta=1$. The inequality (1.1) becomes $|p(z)-1|<|p(z)+1|$, that is

Re, $p(z)>0$. Because $p(0)=1$, so $p(z) \prec \frac{1+z}{1-z}=p_{1,1}(z)$.

Thus for any $0 \leq \lambda \leq 1,0<\beta \leq 1$, we have proved (2.1).

3. THE INTEGRAL MEANS OF FUNCTIONS IN $\mathrm{S}_{\lambda}(\alpha, \beta)$.

We first state some lemmas.

LEMMA 3.1 [2]. For any $g, h \in L^{1}[-\pi, \pi]$, the following statements are equivalent:

(a) For every convex non-decreasing function $\Phi$ on $(-\infty, \infty)$,

(b) For every $t \in(-\infty, \infty)$,

$$
\int_{-\pi}^{\pi} \Phi(g(x)) d x \leq \int_{-\pi}^{\pi} \Phi(h(x)) d x .
$$

$$
\int_{-\pi}^{\pi}(g(x)-t)+d x \leq \int_{-\pi}^{\pi}(h(x)-t)+d x .
$$

(c) $g^{*}(\theta) \leq h^{*}(\theta),(0 \leq \theta \leq \pi)$.

LEMMA 3.2 [2]. If $g, h$ are real integrable functions on $[-\pi, \pi]$, then $(g+h)^{*}(\theta) \leq g^{*}(\theta)+h^{*}(\theta)$ $(0 \leq \theta \leq \pi)$, with equality holding if and only if $g, h$ are symmetric decreasing arrangement functions.

The definitions of $u^{*}(x)$ and the symmetric decreasing arrangement function can be found in [2].

LEMMA 3.3 [3]. Let $\Phi(t)$ be a convex increasing function, if $g(z) \prec f(z)$ in $D$, then

$$
\int_{-\pi}^{\pi} \Phi\left(\left|g\left(r e^{i \theta}\right)\right|\right) d \theta \leq \int_{-\pi}^{\pi} \Phi\left(\left|f\left(r e^{i \theta}\right)\right|\right) d \theta \quad(0<r<1)
$$

and if $u(z)$ is a harmonic function in $D, v(z)=u(\omega(z))$, where $\omega(z)$ is analytic in $D$, $\omega(0)=0,|\omega(z)|<1$, then

$$
\int_{-\pi}^{\pi} \Phi\left( \pm v\left(r e^{i \theta}\right)\right) d \theta \leq \int_{-\pi}^{\pi} \Phi\left( \pm u\left(r e^{i \theta}\right)\right) d \theta \quad(0<r<1) .
$$

When $f(z)$ is not a constant, the equality in (3.1) holds if and only if $\omega(z)=e^{i \theta_{z}}$ or $\Phi(u)=a \log u+b(a<0)$.

Let

$$
k_{\alpha}(z)=\frac{z}{(1-z)^{2(1-\alpha)}}
$$


it is well known that $k_{\alpha}(z) \in S^{*}(\alpha)$. For any $g(z) \in S^{*}(\alpha)$, we have

$$
g(z)=z \exp \left\{2(1-\alpha) \int_{|x|=1} \log \frac{1}{1-x z} d \mu(x)\right\},
$$

so we can easily obtain

$$
\frac{g(z)}{z} \prec \frac{1}{(1-z)^{2(1-\alpha)}}=\frac{k_{\alpha}(z)}{z} .
$$

THEOREM 3.1. If $f(z) \in S_{\lambda}(\alpha, \beta), F_{x}(z)=e^{-i x} k_{\alpha}\left(e^{i x} z\right) \cdot p_{\beta, \lambda}\left(e^{i x} z\right), \Phi(t)$ is a convex nondecreasing function on $(-\infty, \infty)$, then

$$
\int_{-\pi}^{\pi} \Phi\left( \pm \log \frac{\left|f\left(r e^{i \theta}\right)\right|}{r}\right) d \theta \leq \int_{-\pi}^{\pi} \Phi\left( \pm \log \frac{\left|F_{o}\left(r e^{i \theta}\right)\right|}{r}\right) d \theta \quad(0<r<1) .
$$

For a strictly convex function $\Phi$, the equality holds only for $f(z)=F_{x}(z)$.

PROOF. From the definition of $S_{\lambda}(\alpha, \beta)$, we know there exists a function $g(z) \in S^{*}(\alpha)$ such that the inequality (1.1) holds. So we have, from Theorem 2.1

$$
p(z)=\frac{f(z)}{g(z)} \prec \frac{1+\beta z}{1-\beta \lambda z}=P_{\beta, \lambda}(z)
$$

Thus

$$
\int_{-\pi}^{\pi} \Phi\left( \pm \log \left|p\left(r e^{i \theta}\right)\right|\right) d \theta \leq \int_{-\pi}^{\pi} \Phi\left( \pm \log \left|p_{\beta, \lambda}\left(r e^{i \theta}\right)\right|\right) d \theta, \quad \text { by Lemma 3.3. }
$$

Then from Lemma 3.1 we have

$$
\left(\log \left|p\left(r e^{i \theta}\right)\right|\right)^{*} \leq\left(\log \left|p_{\beta, \lambda}\left(r e^{i \theta}\right)\right|\right)^{*} .
$$

On the other hand, because $\frac{f(z)}{z}=p(z) \cdot \frac{g(z)}{z}$, we have, by Lemma 3.2,

$$
\left(\log \frac{\left|f\left(r e^{i \theta}\right)\right|}{r}\right)^{*} \leq\left(\log \left|p\left(r e^{i \theta}\right)\right|\right)^{*}+\left(\log \frac{\left|g\left(r e^{i \theta}\right)\right|}{r}\right)^{*}
$$

Using (3.3) and Lemmas 3.3 and 3.1, we can easily get

So we obtain

$$
\left(\log \frac{\left|g\left(r e^{i \theta}\right)\right|}{r}\right)^{*} \leq\left(\log \frac{\left|k_{\alpha}\left(r e^{i \theta}\right)\right|}{r}\right)^{*}
$$

$$
\left(\log \frac{\left|f\left(r e^{i \theta}\right)\right|}{r}\right)^{*} \leq\left(\log \left|p_{\beta, \lambda}\left(r e^{i \theta}\right)\right|\right)^{*}+\left(\log \frac{\left|k_{\alpha}\left(r e^{i \theta}\right)\right|}{r}\right)^{*} .
$$

By evaluation we know $\log \left|p_{\beta, \lambda}\left(r e^{i \theta}\right)\right|$ and $\log \frac{\left|k_{\alpha}\left(r e^{i \theta}\right)\right|}{r}$ are symmetric decreasing arrangement functions, so again from Lemma 3.2 we have

$$
\left(\log \frac{\left|f\left(r e^{i \theta}\right)\right|}{r}\right)^{*} \leq\left(\log \left|p_{\beta, \lambda}\left(r e^{i \theta}\right) \cdot \frac{k_{\alpha}\left(r e^{i \theta}\right)}{r}\right|\right)^{*}=\left(\log \frac{\left|F_{o}\left(r e^{i \theta}\right)\right|}{r}\right)^{*}
$$

Finally we obtain, by Lemma 3.1,

$$
\int_{-\pi}^{\pi} \Phi\left(\log \frac{\left|f\left(r e^{i \theta}\right)\right|}{r}\right) d \theta \leq \int_{-\pi}^{\pi} \Phi\left(\log \frac{\left|F_{o}\left(r e^{i \theta}\right)\right|}{r}\right) d \theta
$$

We can similarly prove the case of negative sign. The condition of the equality can easily be obtained.

THEOREM 3.2. Let $f(z) \in S_{\lambda}(\alpha, \beta)$, then for $p>0$ we have

$$
\int_{-\pi}^{\pi}\left|f\left(r e^{i \theta}\right)\right|^{p} d \theta \leq \int_{-\pi}^{\pi}\left|k_{\alpha}\left(r e^{i \theta}\right) p_{\beta, \lambda}\left(r e^{i \theta}\right)\right|^{p} d \theta \quad(0<r<1)
$$

and 


$$
\int_{-\pi}^{\pi}\left|f\left(r e^{i \theta}\right)\right|^{-p} d \theta \leq \int_{-\pi}^{\pi}\left|k_{\alpha}\left(r e^{i \theta}\right) p_{\beta, \lambda}\left(r e^{i \theta}\right)\right|^{-p} d \theta \quad(0<r<1)
$$

where the equality holds only for $f(z)=\frac{1}{x} k_{\alpha}(x z) p_{\beta, \lambda}(x z),|x|=1$.

PROOF. We only need let $\Phi(t)=e^{p t}$ in Theorem 3.1.

COROLLARY 3.1. If $f(z) \in S_{\lambda}(\alpha, \beta)$, then we have the following sharp inequality:

$$
\frac{r(1-\beta r)}{(1+r)^{2(1-\alpha)}(1+\beta \lambda r)} \leq|f(z)| \leq \frac{r(1+\beta r)}{(1-r)^{2(1-\alpha)}(1-\beta \lambda r)} \quad(|z|=r) .
$$

PROOF. Take $p$-th root in both sides of (3.5) and (3.6), and let $p \rightarrow \infty$, we can get inequality (3.7).

COROLLARY 3.2. If $f(z) \in S_{\lambda}(\alpha, \beta)$, then we have $f(D) \supset\{w:|w|<d(\alpha, \beta, \lambda)\}$, where

cannot be replaced by any larger number.

$$
d(\alpha, \beta, \lambda)=\frac{1-\beta}{2^{2(1-\alpha)}(1+\beta \lambda)}
$$

PROOF. We can easily know $f(z)$ is univalent in $D$ from the definition of $S_{\lambda}(\alpha, \beta)$, so

$$
\operatorname{dist}(0, \partial f(D))=\lim _{|z| \rightarrow 1} \inf |f(z)| \geq \lim _{|z| \rightarrow 1} \frac{|z|(1-\beta|z|)}{(1+|z|)^{2(1-\alpha)}(1+\beta \lambda|z|)}=\frac{1-\beta}{2^{2(1-\alpha)}(1+\beta \lambda)} \text {. }
$$

The sharpness can bee seen from the function $\frac{z(1+\beta z)}{(1-z)^{2(1-\alpha)}(1-\beta \lambda z)} \in S_{\lambda}(\alpha, \beta)$.

4. A SUBCLASS $\in S_{\lambda}(\alpha, \beta)$.

Let $g(z)=z$, we obtain a subclass $\in S_{\lambda}(\alpha, \beta)$, we denote it by $S_{\lambda}(\beta)$. Corresponding to $(2.1)$, for the class $S_{\lambda}(\beta)$, we have the following subordination:

$$
\frac{f(z)}{z} \prec \frac{1+\beta z}{1-\beta \lambda z}=p_{\beta, \lambda}(z) .
$$

Thus for $S_{\lambda}(\beta)$ we have

THEOREM 4.1. Let $f(z) \in S_{\lambda}(\beta), \Phi(t)$ is a convex non-decreasing function on $(-\infty, \infty)$, then

$$
\int_{-\pi}^{\pi} \Phi\left( \pm \log \frac{\left|f\left(r e^{i \theta}\right)\right|}{r}\right) d \theta \leq \int_{-\pi}^{\pi} \Phi\left( \pm \log \left|\frac{1+\beta r e^{i \theta}}{1-\beta \lambda r e^{i \theta}}\right|\right) d \theta \quad(0<r<1) .
$$

For a strictly convex function $\Phi$, the equality holds only for function $f(z)=z p_{\beta, \lambda}(x z),|x|=1$

If we use subordination (4.1) and Lemma 3.3, we can obtain the following:

THEOREM 4.2. Let $f(z) \in S_{\lambda}(\beta), \Phi(t)$ is a convex non-decreasing function on $(-\infty, \infty)$, then

(a) $\int_{-\pi}^{\pi} \Phi\left(\frac{\left|f\left(r e^{i \theta}\right)\right|}{r}\right) d \theta \leq \int_{-\pi}^{\pi} \Phi\left(\left|\frac{1+\beta r e^{i \theta}}{1-\beta \lambda r e^{i \theta}}\right|\right) d \theta$,

(b) $\quad \int_{-\pi}^{\pi} \Phi\left(\left|\log \frac{f\left(r e^{i \theta}\right)}{r e^{i \theta}}\right|\right) d \theta \leq \int_{-\pi}^{\pi} \Phi\left(\left|\log \frac{1+\beta r e^{i \theta}}{1-\beta \lambda r e^{i \theta}}\right|\right) d \theta$,

(c) $\int_{-\pi}^{\pi} \Phi\left( \pm \arg \frac{f\left(r e^{i \theta}\right)}{r e^{i \theta}}\right) d \theta \leq \int_{-\pi}^{\pi} \Phi\left(\arg \frac{1+\beta r e^{i \theta}}{1-\beta \lambda r e^{i \theta}}\right) d \theta$.

For a strictly convex function $\Phi$, the equality holds only for $f(z)=z p_{\beta, \lambda}(x z),|x|=1$. From (4.5) we obtain the rotation theorem of $S_{\lambda}(\beta)$.

COROLLARY 4.1. Let $f(z) \in S_{\lambda}(\beta)$, then for $|z|=r<1$ we have the following sharp inequality:

$$
\left|\arg \frac{f(z)}{z}\right| \leq \arcsin \frac{\beta(1+\lambda) r}{1+\lambda \beta^{2} r^{2}} .
$$


PROOF. If we take

in (4.5), we have

$$
\Phi(t)= \begin{cases}t^{2 n} & , \quad t \geq 0 \\ 0 & , \quad t<0\end{cases}
$$

$$
\int_{0}^{2 \pi}\left|a r g+\frac{f\left(r e^{i \theta}\right)}{r e^{i \theta}}\right|^{2 n} d \theta \leq \int_{0}^{2 \pi}\left|a r g+\frac{1+\beta r e^{i \theta}}{1-\beta \lambda r e^{i \theta}}\right|^{2 n} d \theta .
$$

Take the $2 n$-th root in both sides of this inequality and let $n \rightarrow \infty$, we get

$$
\max _{-\pi \leq \theta \leq \pi} \arg +\frac{f\left(r e^{i \theta}\right)}{r e^{i \theta}} \leq \max _{-\pi \leq \theta \leq \pi} \arg ^{+} \frac{1+\beta r e^{i \theta}}{1-\beta \lambda r e^{i \theta}}=\arcsin \frac{\beta(1+\lambda) r}{1+\lambda \beta^{2} r^{2}} .
$$

This implies

Similarly, we have

$$
\arg +\frac{f\left(r e^{i \theta}\right)}{r e^{i \theta}} \leq \arcsin \frac{\beta(1+\lambda) r}{1+\lambda \beta^{2} r^{2}}
$$

$$
\arg -\frac{f\left(r e^{i \theta}\right)}{r e^{i \theta}} \leq \arcsin \frac{\beta(1+\lambda) r}{1+\lambda \beta^{2} r^{2}}
$$

So for any $f(z) \in S_{\lambda}(\beta)$, we have

$$
\left|\arg \frac{f(z)}{z}\right| \leq \arcsin \frac{\beta(1+\lambda) r}{1+\lambda \beta^{2} r^{2}} \quad(|z|=r<1),
$$

where they equality holds only for $f(z)=z p_{\beta, \lambda}(x z),|x|=1$. The proof of Corollary 4.1 is complete.

From the univalence of $f(z)$ we know $\frac{f(z)}{z} \neq 0$, so we can define a single-valued and analytic branch of $\log \frac{f(z)}{z}$. Let

then we have:

$$
g(z)=\log \frac{f(z)}{z}=\sum_{n=1}^{\infty} \gamma_{n} z^{n}
$$

COROLLARY 4.2. Let $f(z) \in S_{\lambda}(\beta)$, then we have

$$
\sum_{n=1}^{\infty}\left|\gamma_{n}\right|^{2} \leq \sum_{n=1}^{\infty} \frac{\left(\beta^{n} \lambda^{n}+(-1)^{n-1} \beta^{n}\right)^{2}}{n^{2}}
$$

where the inequality holds only for $f(z)=z p_{\beta, \lambda}(x z),|x|=1$.

PROOF. Let

Take $\Phi(t)=t^{2}$ in (4.4), we have

$$
G(z)=\log \frac{1+\beta z}{1-\beta \lambda z}=\sum_{n=1}^{\infty} \frac{\beta^{n} \lambda^{n}+(-1)^{n-1} \beta^{n}}{n} z^{n} .
$$

that is,

$$
\frac{1}{2 \pi} \int_{0}^{2 \pi}\left|g\left(r e^{i \theta}\right)\right|^{2} d \theta \leq \frac{1}{2 \pi} \int_{0}^{2 \pi}\left|G\left(r e^{i \theta}\right)\right|^{2} d \theta
$$

$$
\sum_{n=1}^{\infty}\left|\lambda_{n}\right|^{2} r^{2 n} \leq \sum_{n=1}^{\infty} \frac{\left(\beta^{n} \lambda^{n}+(-1)^{n-1} \beta^{n}\right)^{2}}{n^{2}} r^{2 n},
$$

let $r \rightarrow 1$, we obtain the inequality we need to prove.

REMARK. Let $\lambda=\beta=1$ in Corollary 4.2, that is $f(z) \in S_{1}(1)$, i.e., $\operatorname{Re}(f(z) / z)>0$.

Inequality (4.6) becomes

This inequality is sharp.

$$
\sum_{n=1}^{\infty}\left|\gamma_{n}\right|^{2} \leq \sum_{n=1}^{\infty} \frac{4}{(2 n-1)^{2}}=\frac{\pi^{2}}{2} .
$$


Finally, we consider the initial coefficients of $f(z) \in S_{\lambda}(\beta)$.

LEMMA 4.1. If $f^{\prime}(0)=F^{\prime}(0)=1$ and they satisfy the following equality

$$
\frac{(1-\beta)-(1+\beta \lambda) f(z) / z}{(1-\beta \lambda) f(z) / z-(1+\beta)}=\frac{F(z)}{z},
$$

then $f(z) \in S_{\lambda}(\beta)$ if and only if $F(z) \in S_{1}(1)$, i.e., $\operatorname{Re}(F(z) / z)>0$.

PROOF. Let $f(z) \in S_{\lambda}(\beta)$, then $p(z)=\frac{f(z)}{z} \prec \frac{1+\beta z}{1-\beta \lambda z}=p_{\beta, \lambda}(z)$, so $p(D) \subset p_{\beta, \lambda}(D)=D_{1}$ where $D_{1}$ is a disk which diameter is $\left(\frac{1-\beta}{1+\beta \lambda}, \frac{1+\beta}{1-\beta \lambda}\right)$, thus

$$
(1-\beta \lambda) p(z)-(1+\beta) \neq 0 .
$$

From this we know $F(z)$ is analytic in $D$. And $F(0)=0$ because of $p(0)=1$. On the other hand, the function

$$
\frac{(1-\beta)-(1+\beta \lambda) w}{(1-\beta \lambda) w-(1+\beta)}
$$

maps $D_{1}$ onto the right half plane, so we have $\left.\operatorname{Re}(\overline{F(z)}) \dot{z}\right)>0(\dot{z} \in \bar{D})$, i.e., $\overline{F(z)} \in \dot{S_{1}}(\hat{1})$.

We can prove the opposite result similarly.

THEOREM 4.3. Let $f(z)=z+\sum_{n=2}^{\infty} a_{n} z^{n} \in S_{\lambda}(\beta)$, then for real number $\mu$ we have the sharp estimates:

$$
\left|a_{3}-\mu a_{2}^{2}\right| \leq\left\{\begin{array}{lc}
\beta(1+\lambda)(\beta \lambda-\mu(\beta+\beta \lambda)), & \left|a_{2}\right| \leq \beta(1+\lambda) \\
\beta(1+\lambda), & -\frac{1-\beta \lambda}{\beta+\beta \lambda}<\mu<\frac{1+\beta \lambda}{\beta+\beta \lambda} \\
\beta(1+\lambda)(\mu(\beta+\beta \lambda)-\beta \lambda), & \mu>\frac{1+\beta \lambda}{\beta+\beta \lambda}
\end{array}\right.
$$

PROOF. Because $f(z) \in S_{\lambda}(\beta)$, then $F(z)$ defined by (4.7) belongs to $S_{1}(1)$, i.e., $\operatorname{Re}(F(z) / z)>0$, so there exists an analytic function $p(z)$ satisfying $p(z)=1+\sum_{n=1}^{\infty} p_{n} z^{n}, \operatorname{Rep}(z)>0$ such that

$$
\frac{F(z)}{z}=p(z)=1+\sum_{n=1}^{\infty} p_{n} z^{n}
$$

Substituting it into (4.7) and comparing the coefficients of both sides of (4.7), we have

$$
a_{2}=\frac{1}{2}(\beta+\beta \lambda) p_{1}, \quad a_{3}-\mu a_{2}^{2}=\frac{1}{2}(\beta+\beta \lambda)\left\{p_{2}-\frac{1}{2}((1-\beta \lambda)+\mu(\beta+\beta \lambda)) p_{1}^{2}\right\} .
$$

It is well known that $\left|p_{n}\right| \leq 2(n=1,2, \cdots \cdots)$

$$
\left|p_{2}-\mu p_{1}^{2}\right| \leq\left\{\begin{array}{ll}
2(1-2 \mu), & \mu \leq 0 \\
2 & , \quad 0<\mu<1 . \\
2(2 \mu-1), & \mu \geq 1
\end{array} .\right.
$$

So we proved the results. Its easy to know the function $f(z)=\frac{z(1+\beta e z)}{1-\beta \lambda e z},(|e|=1)$

attains the equalities in (4.8), (4.9) and (4.11), and the function $f(z)=\frac{z\left(1+\beta e z^{2}\right)}{1-\beta \lambda e z^{2}},(|\varepsilon|=1)$ attains the inequality in (4.10).

\section{REFERENCES}

1. OWA, S., A remark on certain classes of analytic functions, Math. Japon. 28 (1983), 15-20.

2. BAERSTEIN, A., Integral means, univalent functions and circular symmetrization, Acta Math. 133 (1974), 139-169.

3. CHEN, H., Integral means of the subordinate class, Acta Math. Sinica 6 (1990), 739-756.

4. POMMERENKE, CH., Univalent Functions, Göttingen, 1975. 


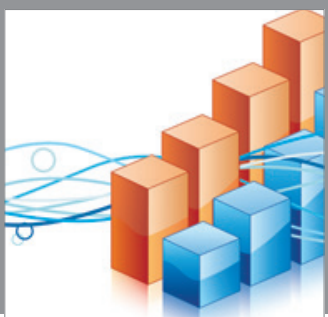

Advances in

Operations Research

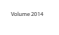

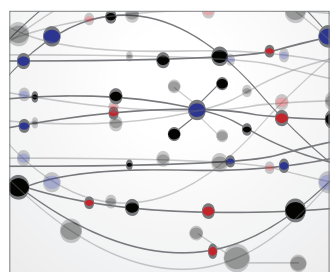

\section{The Scientific} World Journal
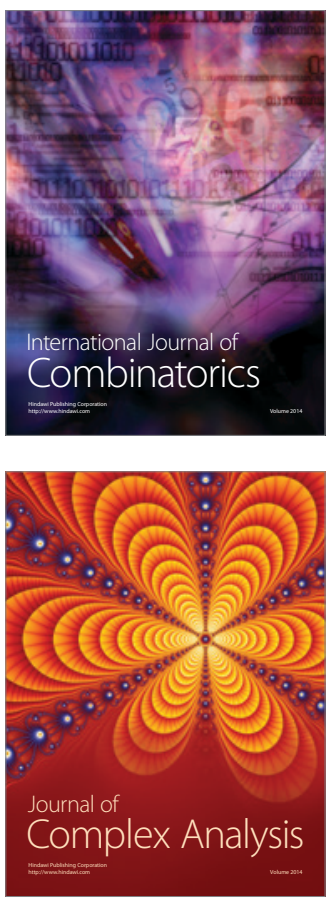

International Journal of

Mathematics and

Mathematical

Sciences
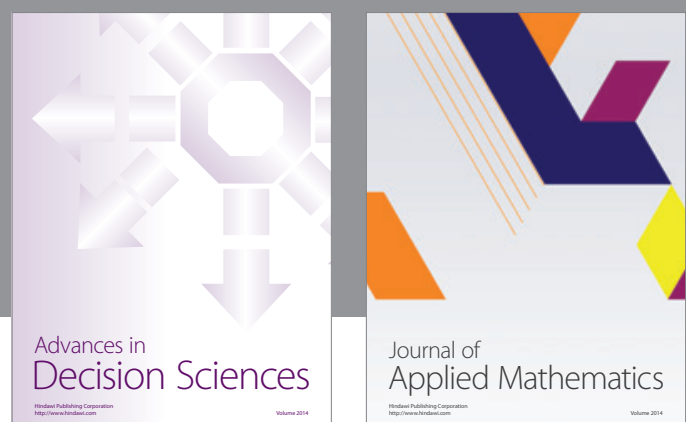

Journal of

Applied Mathematics
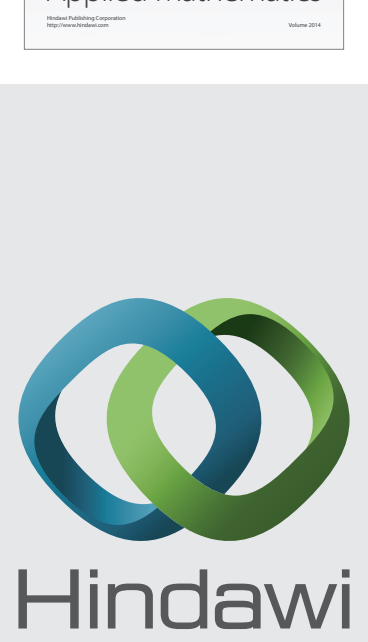

Submit your manuscripts at http://www.hindawi.com
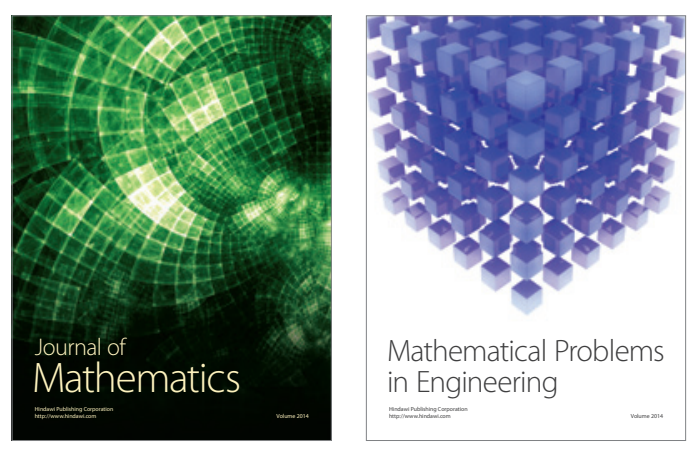

Mathematical Problems in Engineering
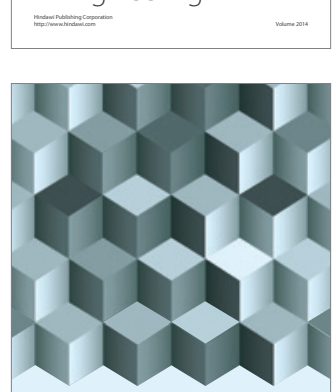

Journal of

Function Spaces
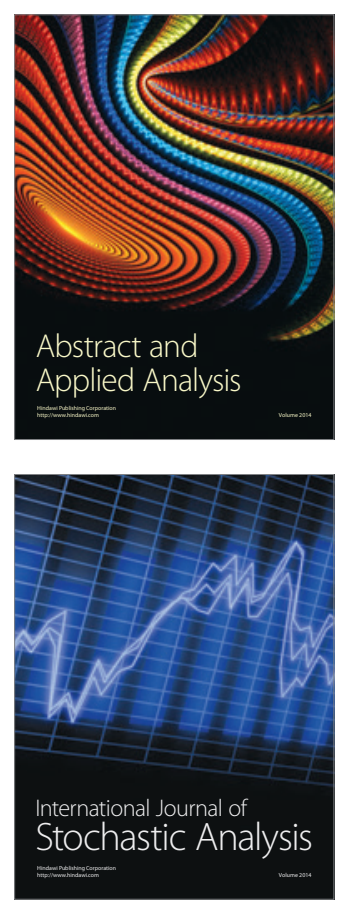

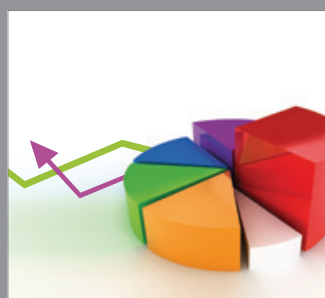

ournal of

Probability and Statistics

Promensencen
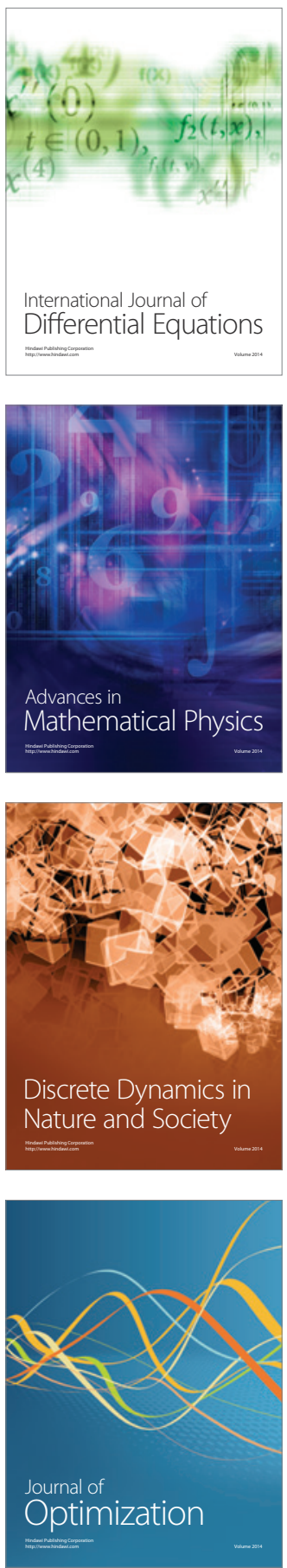